\title{
Network models of dementia progression: the way forward?
}

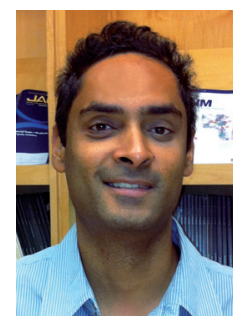

Ashish Raj

Department of Radiology, Weill Medical College of Cornell University, 515 E 71 St, Suite S123, New York,

NY 10044, USA = Tel. : +1 2127461280 = Fax: +1 2127464189 m ashish@med.cornell.edu

Recent breakthroughs in neurodegenerative research are beginning to change our understanding of the causes and effects of dementias like Alzheimer's Disease (AD). These diseases are classically considered as entirely separate disorders, each with its own etiology, progression mechanism and archetypal patterns of atrophy in the brain. Classic patterns of dementias are now well established from longitudinal MRI [1], and are quantified using advanced volumetric analysis (e.g., Free Surfer [2]). Amyloid deposition, metabolism and atrophy in AD show spatially distinct involvement of the posterior temporal heteromodal network [3], while the behavioral variant of frontotemporal dementia (bvFTD) appears restricted to the orbito-frontal network [4]. It was thought that different brain regions are selectively vulnerable to various dementias as a result of differential metabolic and oxidative stress [5]. Buckner et al. conjectured that early metabolic activity in the default network is somehow later implicated in $\mathrm{AD}$ progression [3].

"... it could be that the observed patterns of disease are simply a mechanical result of the way the disease moves around within the brain network."

New evidence points to a different factor: propagation of the disease along the brain's connectivity network. Thus, it could be that the observed patterns of disease are simply a mechanical result of the way the disease moves around within the brain network. If this network-centric hypothesis is true, it could have important ramifications for diagnosis and prediction of future atrophy of patients. It could also upend conventional understanding of different dementias as separate pathological processes affecting very specific brain regions, and instead force future researchers to treat them as different facets of the same underlying process enacted on the brain's network.

\section{Dementias are caused by misfolded proteins that propagate along brain networks}

Dementias are thought to result from the accumulative effect of various species of misfolded proteins. Neuropathological evidence points to $\mathrm{AD}$, the most common dementia, being a neuronal/synaptic polioencephalopathy with the disease beginning in the mesial temporal gray matter regions with accumulation of misfolded $\beta$-amyloid and tau protein [6]. Tau protein and ubiquitin appear to be implicated in FTD, the second most common dementia [4]. In the case of dementia with Lewy bodies, misfolded forms of $\alpha$-synuclein appear to be the culprit [5]. Parkinson's Disease, a rare disorder that displays aspects of neurodegeneration, involves $\alpha$-synuclein [5]. This large array of proteinopathic agents undergo pathological misfolding via complicated and poorly understood pathways.

There is now, however, mounting evidence that despite the variety and complexity of the implicated proteins, the resulting dementias all share a common propagation mechanism in the brain. It has long been known that Alzheimer's atrophy appears to spread in the brain along extant fiber pathways via secondary Wallerian degeneration, disconnection, loss of signaling, axonal reaction and post-synaptic dendrite retraction [6]. Alzheimer's atrophy spreads from mesial temporal structures like the hippocampus to the parietal regions connected to it, and eventually to the frontal connections, until the entire brain has been affected [1]. All this evidence indicates that progression follows vulnerable fiber pathways rather than by proximity [6,9,10]; this understanding has led to a new conception of neurodegeneration as network diseases, affecting

\section{Keywords}

- Alzheimer's disease

- dementia = graph theory

- network diffusion model

- prion-like progression

\section{Future : Medicine part of}


different parts of the brain differently [3]. These findings led to the network degeneration view that various dementias selectively target distinct intrinsic brain networks $[3,6]$.

"Misfolded proteins can trigger
misfolding of adjacent same-species
proteins, which in turn cascade along
neuronal pathways."

The network view received a shot in the arms by new neuropathological evidence that disease proteins, including $\alpha$-synuclein, $\beta$-amyloid and tau, have the capacity to misfold and march throughout local and then long-range circuits via trans-synaptic spread [11-13]. Misfolded proteins can trigger misfolding of adjacent same-species proteins, which in turn cascade along neuronal pathways. Pathological tau conformers can induce nonfolded tau to adopt pathological conformations, which can propagate from the exterior to interior of a cell [14]. These findings suggest a 'prion-like' mechanism of transmission underlying all dementias [11].

\section{Mathematical models can capture prion-like network progression}

In a recent paper our group mathematically modeled this kind of trans-synaptic transmission by a diffusive mechanism, mediated by the healthy brain's connectivity network [15]. Diffusion, a classic model of random dispersion, is an excellent model for any disease-causing agent (e.g., tau, amyloid or synuclein) whose interneuronal advance fulfills the criterion that the rate of propagation is proportional to concentration level differentials. These models were successful previously in modeling neuronal transport and trans-synaptic movement of neurotransmitters [16]. Therefore, we derived the behavior of this diffusive trans-neuronal propagation on whole-brain structural connectivity networks, obtained from whole-brain tractography of diffusion MRI scans [17]. The nodes of this network correspond to gray matter structures of a labeled brain atlas, and its edges denote the strength of fiber tract connectivity.

Subsequent analysis of this network using graph theoretic algorithms demonstrated that the macroscopic consequences of this kind of diffusive prion-like propagation on the whole-brain healthy network (henceforth called the 'network diffusion model') are surprisingly consistent with known patterns of atrophy in various dementias. Specifically, the model predicted spatially distinct "eigen-modes," which were found to recapitulate known disjoint patterns of several dementias, as well as T1-weighted MRI volumetrics of our AD and FTD subjects. The eigen-modes also recapitulate recent findings of dissociated brain networks selectively targeted by different dementias [3,6]. Prevalence rates predicted by the model strongly agree with published data. To our knowledge, this is the first time that qualitative neuropathological observations have been successfully converted into a fully testable quantitative model that recapitulates well-known but hitherto unexplained segregated domains of various dementias.

\section{Are all dementias facets of the same degenerative mechanism?}

The success of network dynamic models like [15] is especially noteworthy because it provides a systemic explanation of macroscopic dementia patterns using a strictly mechanical microscopic model - without requiring any kind of selective vulnerability, regional specificity or shared functional load. Since our results are the same with even random starting configurations, we concluded that purely network dynamics are sufficient to explain archetypal patterns of regional specificity seen in various dementias [3,6-8]. The network diffusion model thus accommodates a new conception of these diseases that is fully consistent with known findings, but does not require region-specific neuropathy, for example, of mesial temporal origin [8], or selective vulnerability within dissociated functional networks [7]. Since the model makes no distinction between different dementias or proteinopathic carriers, this raises a somewhat unorthodox possibility that diverse degenerative etiologies share a common progression mechanism and might have common macroscopic consequences.

$$
\begin{aligned}
& \text { 6...the macroscopic consequences of } \\
& \text { this kind of diffusive prion-like } \\
& \text { propagation on the whole-brain healthy } \\
& \text { network ... are surprisingly consistent with } \\
& \text { known patterns of atrophy in various } \\
& \text { dementias." }
\end{aligned}
$$

These ideas are generally consistent with recent investigations. The idea that proteinopathic carriers with varied etiology can have a shared progression mechanism via 'permissible templating' was first raised by Hardy [18]. The etiology of neurodegeneration and the effect of individual misfolding proteins are known to be diverse and mutually interactive $[11,12]$. 
Joint histopathological/morphometric studies suggest that the specific biochemical properties of the prion-like agent may be inconsequential for the macroscopic and chronic clinical manifestation of disease. The spatial distribution of $\beta$-amyloid pathology in $\mathrm{AD}$ is poorly correlated with whole-brain atrophy patterns [19], while tau is well-correlated. However, neither tau nor $\beta$-amyloid are specific to $A D$ and are found in semantic dementia, FTD [20], dementia with Lewy bodies and posterior cortical atrophy [21] among others. Pittsburgh compound B-positive binding to A- $\beta$ and plaques were observed in $25-45 \%$ of cognitively normal older subjects in postmortem autopsy studies [21]. bvFTD accommodates an array of pathological correlates, including $\alpha$-synuclein, tau, ubiquitin, TDP-43 and Lewy bodies [20]. Pereira et al. found that clinical variants of bvFTD, but not histologic variants, correlated with regional atrophy, and no volumetric difference was found between tau and ubiquitin bvFTD pathology regardless of clinical subtype [20].

Excellent corroboration of these ideas was provided by the Seeley laboratory [6,22], who show that resting-state functional connectivity of healthy brain regions is correlated with vulnerability to atrophy in five different neurodegenerative diseases. They conclude that these findings are more compatible with a transneuronal model of pathology spread compared with other hypotheses.

\section{Towards a personalized weather radar map of dementia?}

Further validation of the network diffusion model can have a significant impact on clinical practice. First, the model provides a set of basis functions upon which complicated and clinically uninterpretable whole-brain atrophy data can be projected, thus summarizing the patients' dementia state into only two or three numbers, which may be used for differential diagnosis (see Figure 7 in [15]). These summary projections can act as effective clinical biomarkers for unsupervised, automated and regionally unbiased differential diagnosis of various dementias. This approach could be especially helpful in cases of mixed dementia, where classical region-based atrophy descriptors might prove unsatisfactory.

However, the most important clinical application of this model could well be in the prediction of cognitive decline. Starting from baseline MRI volumetrics for estimation of model parameters, the model can subsequently be used to predict future atrophy of an individual subject. This will allow a neurologist to predict what the patient's neuroanatomic and therefore cognitive state will be at any given point in the future. This is not dissimilar to a weather radar map, which provides an hourly forecast of the coming weather pattern in a geographical area. Knowledge of what the future holds will allow patients to make informed choices regarding their lifestyle and therapeutic interventions. Although all this seems rather futuristic, these are real possibilities, the successful adoption of which transform the long-term management and care of dementias.

\section{Financial \& competing interests disclosure \\ This research was supported in part by NIH grants $R 01$ NS075425, F32 EB012404-01 and P41 RR023953- \\ 02. The author has no other relevant affiliations or financial involvement with any organization or entity with a financial interest in or financial conflict with the subject matter or materials discussed in the manuscript apart from those disclosed. \\ No writing assistance was utilized in the production of this manuscript.}

\section{References}

1. Thompson PM, Hayashi KM, de Zubicaray $\mathrm{G}$ et al. Dynamics of gray matter loss in Alzheimer's disease. J. Neurosci. 23(3), 994-1005 (2003).

2. Fischl B, Salat DH, Busa E et al. Whole brain segmentation: automated labeling of neuroanatomical structures in the human brain. Neuron 33(3), 341-355 (2002).

3. Buckner RL, Snyder AZ, Shannon BJ et al. Molecular, structural, and functional characterization of Alzheimer's disease: evidence for a relationship between default activity, amyloid, and memory. J. Neurosci. 25(34), 7709-7717 (2005).
4. Whitwell JL, Jack CR, Senjem ML et al. MRI correlates of protein deposition and disease severity in postmortem frontotemporal lobar degeneration. Neurodegener. Dis. 6, 106-117 (2009).

5. Pacheco C, Aguayo LG, Opazo C. An extracellular mechanism that can explain the neurotoxic effects of $\alpha$-synuclein aggregates in the brain. Front Physiol. 3, 297 (2012).

6. Seeley WW, Crawford RK, Zhou J, Miller BL, Greicius MD. Neurodegenerative diseases target large-scale human brain networks. Neuron 62(1), 42-52 (2009).

7. Saxena S, Caroni P. Selective neuronal vulnerability in neurodegenerative diseases: from stressor thresholds to degeneration. Neuron 71(1), 35-48 (2011).

8. Braak H, Del Tredici K, Schultz C, Braak E. Vulnerability of select neuronal types to Alzheimer's disease. Ann. NY Acad. Sci. 924, 53-61 (2000).

9. Englund E, Brun A, Alling C. White matter changes in dementia of Alzheimer's type. Biochemical and neuropathological correlates. Brain 111(Pt 6), 1425-1439 (1988).

10. Villain N, Desgranges B, Viader F et al. Relationships between hippocampal atrophy, white matter disruption, and gray matter hypometabolism in Alzheimer's disease. J. Neurosci. 28(24), 6174-6181 (2008). 
11. Frost B, Diamond MI. Prion-like mechanisms in neurodegenerative diseases. Nat. Rev. Neurosci. 11(3), 155-159 (2010).

12. Palop JJ, Chin J, Mucke L. A network dysfunction perspective on neurodegenerative diseases. Nature 443(7113), 768-773 (2006).

13. Liu L, Drouet V, Wu JW et al. Trans-synaptic spread of tau pathology in vivo. PLoS ONE 7(2), e31302 (2012).

14. Frost B, Ollesch J, Wille H, Diamond MI. Conformational diversity of wild-type tau fibrils specified by templated conformation change. J. Biol. Chem. 284(6), 3546-3551 (2009).

15. Raj A, Kuceyeski A, Weiner M. A network diffusion model of disease progression in dementia. Neuron 73(6), 1204-1215 (2012).
16. Barreda JL, Zhou HX. A solvable model for the diffusion and reaction of neurotransmitters in a synaptic junction. BMC Biophys. 4, 5 (2011).

17. Lo CY, Wang PN, Chou KH et al. Diffusion tensor tractography reveals abnormal topological organization in structural cortical networks in Alzheimer's disease. J. Neurosci. 30(50), 16876-16885 (2010).

18. Hardy J. Expression of normal sequence pathogenic proteins for neurodegenerative disease contributes to disease risk: 'permissive templating' as a general mechanism underlying neurodegeneration. Biochem. Soc. Trans. 33(Pt 4), 578-581 (2005).

19. Rabinovici GD, Furst AJ, Alkalay A et al. Increased metabolic vulnerability in early-onset Alzheimer's disease is not related to amyloid burden. Brain 133(Pt 2), 512-528 (2010).

20. Pereira JMS, Williams GB, Acosta-Cabronero $\mathrm{J}$ et al. Atrophy patterns in histologic vs clinical groupings of frontotemporal lobar degeneration. Neurology 72(19), 1653-1660 (2009).

21. Rabinovici GD, Jagust WJ. Amyloid imaging in aging and dementia: testing the amyloid hypothesis in vivo. Behav. Neurol. 21(1), 117-128 (2009).

22. Zhou J, Gennatas ED, Kramer JH, Miller BL, Seeley WW. Predicting regional neurodegeneration from the healthy brain functional connectome. Neuron 73(6), 1216-1227 (2012). 